\title{
Doubly even orientable closed 2-cell embeddings of the complete graph
}

\author{
Mike J. Grannell \\ Department of Mathematics and Statistics \\ The Open University \\ Walton Hall \\ Milton Keynes MK7 6AA, United Kingdom \\ $\mathrm{m} \cdot \mathrm{j} \cdot \mathrm{grannell@open.ac.uk}$ \\ Thomas A. McCourt \\ Heilbronn Institute for Mathematical Research \\ School of Mathematics \\ University of Bristol \\ University Walk \\ Bristol BS1 1TW, United Kingdom \\ tom.a.mccourt@gmail.com
}

Submitted: Mar 6, 2013; Accepted: Jan 23, 2014; Published: Feb 7, 2014

Mathematics Subject Classifications: 05C10, 05C51

\begin{abstract}
For all $m \geqslant 1$ and $k \geqslant 2$, we construct closed 2-cell embeddings of the complete graph $K_{8 k m+4 k+1}$ with faces of size $4 k$ in orientable surfaces. Moreover, we show that when $k \geqslant 3$ there are at least $(2 m-1) ! / 2(2 m+1)=2^{2 m \log _{2} m-\mathrm{O}(m)}$ nonisomorphic embeddings of this type. We also show that when $k=2$ there are at least $\frac{1}{4} \pi^{\frac{1}{2}} m^{-\frac{5}{4}}\left(\frac{4 m}{e^{2}}\right)^{\sqrt{m}}(1-\mathrm{o}(1))$ nonisomorphic embeddings of this type.
\end{abstract}

\section{Introduction and motivation}

Consider an embedding of a simple graph $G$ in an orientable surface. If each of the faces of the embedding is homeomorphic to an open disk, then the embedding is said to be a 2-cell embedding of $G$. Moreover, if no vertex appears more than once in the facial walk of any given face (that is, all the facial walks are cycles of $G$ ), then the embedding is said to be a closed 2-cell embedding. Hence, if the faces of a 2-cell embedding of a simple graph $G$ with no degree one vertices are all of size less than six, then the embedding is necessarily closed. Unless otherwise stated all the embeddings we discuss will be orientable. 
In this paper we will be interested in closed 2-cell embeddings of the complete graph $K_{n}$ in which all the faces have the same size. We will denote such an embedding, in which all the faces have size $s$ say, as an $s-2 \mathrm{CS}(n)$ embedding (the etymology of this notation being that the faces of such an embedding correspond to a twofold $s$-cycle system of order $n$, an $s-2 \mathrm{CS}(n))$.

To date the only known results on $s-2 \mathrm{CS}(n)$ embeddings either fix $s$ and vary $n$, or vary $s$ but have $n=f(s)$, where $f$ is some fixed linear function. The aim of this paper is to provide $s-2 \mathrm{CS}(n)$ embeddings in which both $s$ and $n$ can vary independently.

In solving the Heawood Map Colouring Conjecture for orientable surfaces of genus greater or equal to one, Youngs [14] and Ringel [13] provide triangulations of the complete graph, $K_{n}$, whenever the obvious congruence conditions $(n \equiv 0,3,4,7(\bmod 12))$ are satisfied. In 2000, for $n=12 s+7$ where $s \equiv 0,1(\bmod 3)$, Bonnington et. al. [2], showed that there are at least $2^{n^{2} / 54-\mathrm{O}(n)}$ nonisomorphic orientable triangulations of $K_{n}$. Since 2000 there have been over half a dozen research papers on constructing nonisomorphic triangulations of the complete graph. Currently the best known lower bounds on the number of nonisomorphic triangulations of $K_{n}$ in either orientable or nonorientable surfaces are of the form $n^{a n^{2}}$ for suitable constants $a>0$, although these bounds have only been established for a sparse set of values of $n[5,7]$.

In [12], Korzhik and Voss constructed $2^{4 m-1}$ nonisomorphic 2-cell orientable embeddings, where all the faces have size four, of the complete graph $K_{8 m+5}$, i.e. quadrangular embeddings. Recently Korzhik [11] improved this result by constructing $2^{2 m \log _{2} m-\mathrm{O}(m)}$ nonisomorphic orientable quadrangular embeddings of $K_{8 m+5}$.

As mentioned earlier, if an embedding of a complete graph is a triangulation or a quadrangulation (that is, all the faces either have size 3 or size 4), the embedding is trivially closed 2-cell and hence is a $s$-2CS(n) embedding for either $s=3$ or $s=4$.

Ellingham and Stephens, in [3] and [4], constructed $n$-2CS $(n)$ embeddings for all $n>5$ in nonorientable surfaces and $n$-2CS $(n)$ embeddings in orientable surfaces for $n=2^{p}+2$ and $p \geqslant 3$. In [8], Griggs and McCourt constructed $n$-2CS(2n+1) embeddings in orientable surfaces for all odd $n \geqslant 3$ and in nonorientable surfaces for all $n \geqslant 4$. By applying Theorem 3.1 of [6] to the results of [5] and [7], a lower bound of the form $n^{a n^{2}}$ on the number of nonisomorphic $n$-2CS $(n)$ embeddings may be obtained for certain values of $n$. For both $n$-2CS $(n)$ and $n$-2CS $(2 n+1)$ embeddings with $n \geqslant 6$, it is non-trivial to ensure that the embeddings are closed 2-cell embeddings.

For an $s-2 \mathrm{CS}(n)$ embedding to exist, a necessary condition is that an $s-2 \mathrm{CS}(n)$ should exist, so $s \leqslant n$ and $s$ must divide $n(n-1)$ [1]. We will construct embeddings for $s=4 k$ where $k \geqslant 2$ and where the corresponding $4 k-2 \mathrm{CS}(n)$ has a cyclic automorphism of order $n$ so that the faces of the embedding appear in orbits of length $n$; a necessary condition for this is that $4 k$ divides $(n-1)$. As we wish to construct orientable embeddings, a quick calculation with Euler's formula yields the condition that $n=8 k m+4 k+1$, for some $m \geqslant 0$.

In Section 3, for all $m, k \geqslant 1$ we construct closed 2-cell embeddings of $K_{8 k m+4 k+1}$ with faces of size $4 k$ in orientable surfaces. In Section 4 we show that, if $k=2$, there are at least $\frac{1}{4} \pi^{\frac{1}{2}} m^{-\frac{5}{4}}\left(\frac{4 m}{e^{2}}\right)^{\sqrt{m}}(1-\mathrm{o}(1))$ such embeddings and, if $k \geqslant 3$, there are at least 
$(2 m-1) ! / 2(2 m+1)=2^{2 m \log _{2} m-\mathrm{O}(m)}$ such embeddings. First however, in Section 2, we will discuss current graphs, the machinery of which we will use to prove the results in Section 3.

\section{Current graphs}

We will construct the embeddings from index 1 current graphs and we assume that the reader is familiar with these techniques. A good overview of current graphs can be found in [10], and we will follow the notation established in that book.

Our construction makes extensive use of the following Theorem due to Gross and Alpert [9] (we state a simpler version of the theorem in which all the edges in question are orientation preserving).

Theorem $1([9])$. Let $e_{1} \ldots e_{d}$ be the rotation at vertex $v$ of the current graph $\langle G \rightarrow S, \mathcal{B}\rangle$, and let $c_{i}$ be the current carried by the direction of the edge $e_{i}$ that has $v$ as its initial vertex. Let $c=c_{1} \ldots c_{d}$, and let $r$ be the order of $c$ in the current group $\mathcal{B}$. Then the derived embedding has $|\mathcal{B}| / r$ faces corresponding to vertex $v$, each of size $r d$, and each of the form

$$
\begin{gathered}
\left(e_{1}, b\right),\left(e_{2}, b c_{1}\right),\left(e_{3}, b c_{1} c_{2}\right), \ldots,\left(e_{d}, b c_{1} c_{2} \ldots c_{d-1}\right),\left(e_{1}, b c\right),\left(e_{2}, b c c_{1}\right),\left(e_{3}, b c c_{1} c_{2}\right), \ldots \\
\ldots,\left(e_{1}, b c^{r}\right)=\left(e_{1}, b\right) .
\end{gathered}
$$

In order for the derived embedding to be that of a complete graph it is sufficient for the current graph to have $(|\mathcal{B}|-1) / 2$ edges and be a one-face embedding in which each edge is labelled with an element of $\mathcal{B} \backslash\{0\}$, such that for each element $i \in \mathcal{B} \backslash\{0\}$ either exactly one edge is labelled $i$ or exactly one edge is labelled $i^{-1}$, i.e. $\langle G \rightarrow S, \mathcal{B}\rangle$ is an index 1 current graph; moreover, if $S$ is an orientable surface, then the derived embedding is also orientable; see [10]. In this paper our current graphs will only have type 0 (untwisted) edges.

Set $n:=8 k m+4 k+1$. For the current graphs that are constructed in this paper we set $\mathcal{B}:=\mathbb{Z}_{n}$, so, from here on, we will write the group operation additively. We make the following observation on index 1 current graphs, which is a direct consequence of Theorem 1 .

Observation 2.1. Suppose that the vertex $v$ has degree $4 k$, rotation $e_{1} \ldots e_{4 k}$, where edge $e_{i}$ carries current $c_{i}$ directed away from $v$, and that $\sum_{i=1}^{4 k} c_{i}=0$. Then the corresponding faces in the derived graph have $4 k$ edges and there are $n$ such faces. Moreover, if the set $\left\{\sum_{1 \leqslant j \leqslant i} c_{j}: 1 \leqslant i \leqslant 4 k\right\}$ has cardinality $4 k$, then the boundary walk of each of these faces is a cycle of length $4 k$.

Thus, in order to construct our desired embedding the construction of a current graph $\left\langle G \rightarrow S, \mathbb{Z}_{n}\right\rangle$ with the following properties would suffice.

(i) The graph $G$ has $2 m+1$ vertices and is regular of degree $4 k$. 
(ii) There is a circuit of the embedding $G \rightarrow S$ in which every edge is traversed exactly once in each direction, i.e. the embedding is an orientable one-face embedding.

(iii) Each edge in $G \rightarrow S$ is assigned a direction and a distinct current from $\mathbb{Z}_{n} \backslash\{0\}$, moreover, if some edge is labelled $i$, then there does not exist an edge labelled $-i$.

(iv) For every vertex $v$, if $e_{1} \ldots e_{4 k}$ is the rotation of the vertex, where edge $e_{i}$ carries current $c_{i}$ directed away from $v$, then $\sum_{i=1}^{4 k} c_{i}=0$ and the set $\left\{\sum_{1 \leqslant j \leqslant i} c_{j}: 1 \leqslant i \leqslant\right.$ $4 k\}$ has cardinality $4 k$.

The graph $G$ that we will employ is the (multi-)graph $2 k C_{2 m+1}$, this graph clearly satisfies Property (i). See Figure 1 for an example with $k=m=2$.

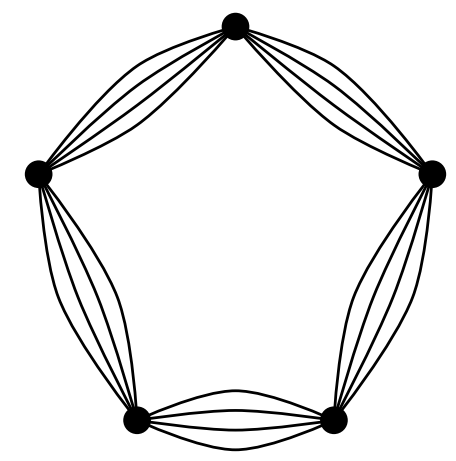

Figure 1: The graph $4 C_{5}$.

\section{Constructing doubly even embeddings}

We will construct a rotation of $2 k C_{2 m+1}$ that induces an orientable one-face embedding (such an embedding satisfies Properties (i) and (ii)). Then we give a current assignment of $2 k C_{2 m+1}$ satisfying Properties (iii) and (iv).

Lemma 3.1. For $m \geqslant 1$, there exists an upper-embedding of $2 C_{2 m+1}$ in an orientable surface.

Proof. Let $V\left(2 C_{2 m+1}\right)=\left\{v_{0}, v_{1}, \ldots, v_{2 m}\right\}$. Denote the two edges between vertices $v_{i}$ and $v_{i+1}$ (where subscripts are taken modulo $2 m+1$ ) as $e_{i}$ and $e_{2 m+1+i}$. Now, we assign the following edge rotations at each vertex 


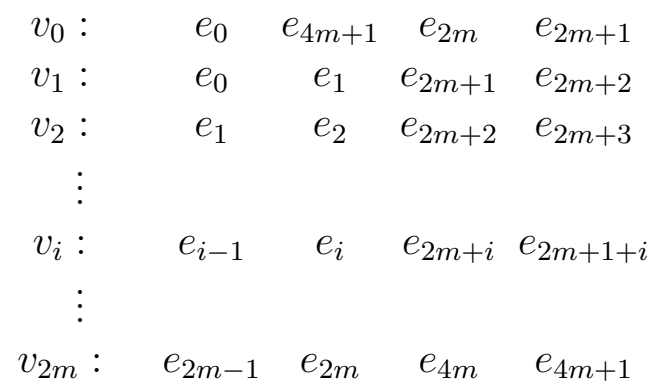

It is easy to check that the embedding described has precisely one facial walk, namely

$$
\begin{gathered}
e_{2 m+1}, e_{2 m+2}, e_{2 m+3}, \ldots, e_{4 m}, e_{4 m+1}, e_{2 m}, e_{4 m}, e_{2 m-2}, e_{4 m-2}, \ldots, e_{2}, e_{2 m+2}, e_{0}, \\
e_{4 m+1}, e_{2 m-1}, e_{4 m-1}, e_{2 m-3}, \ldots, e_{1}, e_{2 m+1}, e_{0}, e_{1}, e_{2}, e_{3}, \ldots, e_{2 m-1}, e_{2 m},
\end{gathered}
$$

and that each edge is traversed precisely once in each direction in this walk. (We have suppressed the vertices from this sequence as they are implicit from the edges.)

Figure 2 shows the one-face embedding when $m=2$ (in the figure the vertices have an anticlockwise orientation).

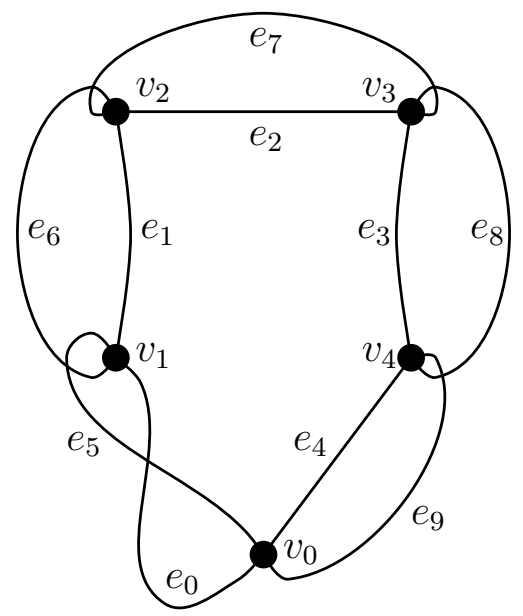

Figure 2: The embedding from Lemma 3.1 when $m=2$.

Lemma 3.2. Suppose we have a graph $G$ and a rotation $D$ of $G$ inducing an orientable one-face embedding of $G$. Suppose that $u$ and $v$ are distinct vertices of $G$ and the edge rotation at $u$ is $D_{u}=\ldots, a, b, \ldots$ and the edge rotation at $v$ is $D_{v}=\ldots, c, d, \ldots$ Then if we add two parallel (type 0 ) edges $f_{1}$ and $f_{2}$ joining $u$ and $v$, and define the rotation $D^{\prime}$ of the obtained graph as $D_{u}^{\prime}=\ldots, a, f_{1}, f_{2}, b, \ldots, D_{v}^{\prime}=\ldots, c, f_{1}, f_{2}, d, \ldots$, and $D_{w}^{\prime}=D_{w}$ for all $w \notin\{u, v\}$, then $D^{\prime}$ induces an orientable one-face embedding.

See Figure 3 for an illustration of Lemma 3.2. 


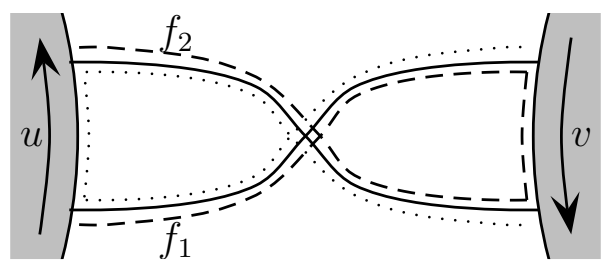

Figure 3: Illustration of Lemma 3.2.

Proof. Without loss of generality the facial walk is of the form

$$
a, u, b, \ldots c, v, d, \ldots
$$

The addition of the edges $f_{1}$ and $f_{2}$ as described in the statement of the lemma yields an embedding with the facial walk

$$
a, u, f_{1}, v, f_{2}, u, b, \ldots c, v, f_{1}, u, f_{2}, v, d, \ldots
$$

Hence, we have an orientable upper-embedding of $G=\left(V(G), E(G) \cup\left\{f_{1}, f_{2}\right\}\right)$.

Theorem 2. For $k, m \geqslant 1$, there exists an orientable $4 k-2 C S(8 k m+4 k+1)$ embedding.

Proof. When $k=1$ the result follows from [12]. So we need only consider $k \geqslant 2$.

For each of the cases below we first construct an upper-embedding of $2 k C_{2 m+1}$ using Lemmas 3.1 and 3.2. Starting with the embedding of $2 C_{2 m+1}$ from Lemma 3.1, for each $0 \leqslant i \leqslant 2 m$, we add the pairs of edges $f_{i}^{2 j-1}, f_{i}^{2 j}$, where $1 \leqslant j \leqslant k-1$, between vertices $v_{i}$ and $v_{i+1}$ (with subscripts taken modulo $2 m+1$ ) using Lemma 3.2 to yield the following edge rotations (the sign of the edge indicates the orientation of the edge, positive for an edge directed into the vertex and negative otherwise).

$$
\begin{array}{ccccccccccccc}
v_{0}: & -e_{0} & e_{4 m+1} & f_{0}^{1} & f_{0}^{2} & \ldots & f_{0}^{2(k-1)} & e_{2 m} & -e_{2 m+1} & -f_{2 m}^{1} & -f_{2 m}^{2} & \ldots & -f_{2 m}^{2(k-1)} \\
v_{1}: & e_{0} & -e_{1} & f_{1}^{1} & f_{1}^{2} & \ldots & f_{1}^{2(k-1)} & e_{2 m+1} & -e_{2 m+2} & -f_{0}^{1} & -f_{0}^{2} & \ldots & -f_{0}^{2(k-1)} \\
v_{2}: & e_{1} & -e_{2} & f_{2}^{1} & f_{2}^{2} & \ldots & f_{2}^{2(k-1)} & e_{2 m+2} & -e_{2 m+3} & -f_{1}^{1} & -f_{1}^{2} & \ldots & -f_{1}^{2(k-1)} \\
\vdots & & & & & & & & & & & & \\
v_{i}: & e_{i-1} & -e_{i} & f_{i}^{1} & f_{i}^{2} & \ldots & f_{i}^{2(k-1)} & e_{2 m+i} & -e_{2 m+1+i} & -f_{i-1}^{1} & -f_{i-1}^{2} & \ldots & -f_{i-1}^{2(k-1)} \\
\vdots & & & & & & & & & & & & \\
v_{2 m}: & e_{2 m-1} & -e_{2 m} & f_{2 m}^{1} & f_{2 m}^{2} & \ldots & f_{2 m}^{2(k-1)} & e_{4 m} & -e_{4 m+1} & -f_{2 m-1}^{1} & -f_{2 m-1}^{2} & \ldots & -f_{2 m-1}^{2(k-1)}
\end{array}
$$

We denote this embedding as $2 k C_{2 m+1} \rightarrow S$. By Lemmas 3.1 and $3.2,2 k C_{2 m+1} \rightarrow S$ is an orientable upper embedding and so Property (ii) is satisfied.

We begin with the case where $k \geqslant 3$. The edges of $2 k C_{2 m+1} \rightarrow S$ are labelled as follows:

$$
\begin{aligned}
& e_{i}: 4 k m-2 k i+2 k, \text { for } 0 \leqslant i \leqslant 2 m ; \\
& e_{i}: 2 k i+2, \text { for } 2 m+1 \leqslant i \leqslant 4 m+1 ;
\end{aligned}
$$


$f_{2 i}^{j}:(-1)^{j-1}(2 k i+j)$, for $0 \leqslant i \leqslant m$ and $1 \leqslant j \leqslant 2(k-1)$; and

$f_{2 i-1}^{j}:(-1)^{j-1}(4 k m-2 k i+2 k+j)$, for $1 \leqslant i \leqslant m$ and $1 \leqslant j \leqslant 2(k-1)$.

Note that these currents (and their negatives) provide each of the elements of $\mathbb{Z}_{n} \backslash\{0\}$ exactly once; hence, Property (iii) is satisfied. This labelling, together with the above edge rotations at the vertices, yields the following series;

$$
\begin{aligned}
v_{0}: \quad(-(4 k m+2 k))+(8 k m+2 k+2)+\left[\sum_{j=1}^{2(k-1)}(-1)^{j-1} j\right] \\
+(2 k)+(-(4 k m+2 k+2))+\left[\sum_{j=1}^{2(k-1)}(-1)^{j}(2 k m+j)\right]
\end{aligned}
$$

and, for $1 \leqslant i \leqslant m$;

$$
\begin{aligned}
& v_{2 i-1}: \quad(4 k m+6 k-4 k i)+(-(4 k m+4 k-4 k i)) \\
&+ {\left[\sum_{j=1}^{2(k-1)}(-1)^{j-1}(4 k m+2 k-2 k i+j)\right]+(4 k m+4 k i-2 k+2) } \\
&+(-(4 k m+4 k i+2))+\left[\sum_{j=1}^{2(k-1)}(-1)^{j}(2 k i-2 k+j)\right] \\
& v_{2 i}: \quad(4 k m+4 k-4 k i)+(-(4 k m+2 k-4 k i))+\left[\sum_{j=1}^{2(k-1)}(-1)^{j-1}(2 k i+j)\right. \\
&+(4 k m+4 k i+2)+(-(4 k m+2 k+4 k i+2)) \\
&+\left[\sum_{j=1}^{2(k-1)}(-1)^{j}(4 k m+2 k-2 k i+j)\right]
\end{aligned}
$$

Hence, these labellings yield the following sequences of partial sums;

$$
\begin{aligned}
v_{0}: & 4 k m+2 k+1,4 k m+2,(4 k m+2+j, 4 k m+2-j)_{j=1}^{k-1}, \\
& 4 k m+k+3,8 k m+3 k+2,(6 k m+3 k+2-j, 8 k m+3 k+2+j)_{j=1}^{k-1}
\end{aligned}
$$

and, for $1 \leqslant i \leqslant m$;

$$
\begin{aligned}
v_{2 i-1}: & 4 k m+6 k-4 k i, 2 k,(4 k m+4 k-2 k i+j, 2 k-j)_{j=1}^{k-1}, 4 k m-k+4 k i+3, \\
& 8 k m+3 k+2,(8 k m+5 k-2 k i+2-j, 8 k m+3 k+2+j)_{j=1}^{k-1} \\
v_{2 i}: \quad & 4 k m+4 k-4 k i, 2 k,(2 k+2 k i+j, 2 k-j)_{j=1}^{k-1}, 4 k m+k+4 k i+3, \\
& 8 k m+3 k+2,(4 k m+k+2 k i+2-j, 8 k m+3 k+2+j)_{j=1}^{k-1} .
\end{aligned}
$$


It is easy to check that, as $k \geqslant 3$, for vertex $v_{l}$, where $0 \leqslant l \leqslant 2 m$, the terms in the sequence of partial sums are all distinct and that the final term is 0; hence Property (iv) holds.

For $k=2$ the labellings given above do not satisfy Property (iv); for example, the partial sums for $v_{0}$ given by $4 k m+2 k+1$ and $4 k m+k+3$ are equal. Instead, when $k=2$, the edges of $2 k C_{2 m+1} \rightarrow S$ may be labelled as follows:

$$
\begin{aligned}
& e_{i}: 8 m-4 i+4, \text { for } 0 \leqslant i \leqslant 2 m ; \\
& e_{i}: 4 i+2, \text { for } 2 m+1 \leqslant i \leqslant 4 m+1 ; \\
& f_{2 i}^{j}:(-1)^{j}(4 i+j), \text { for } 0 \leqslant i \leqslant m \text { and } j=1,2 \text {; and } \\
& f_{2 i-1}^{j}:(-1)^{j}(8 m-4 i+4+j), \text { for } 1 \leqslant i \leqslant m \text { and } j=1,2 .
\end{aligned}
$$

Note that this labelling satisfies Property (iii). This time the labelling yields the following series;

$v_{0}:(-(8 m+4))+(16 m+6)+(-1)+(2)+(4)+(-(8 m+6))+(4 m+1)+(-(4 m+2))$

and, for $1 \leqslant i \leqslant m$

$$
\begin{aligned}
v_{2 i-1}: \quad & (8 m+12-8 i)+(-(8 m+8-8 i))+(-(8 m-4 i+5))+(8 m-4 i+6) \\
& +(8 m+8 i-2)+(-(8 m+8 i+2))+(4 i-3)+(-(4 i-2)) \\
v_{2 i}: \quad & (8 m+8-8 i)+(-(8 m+4-8 i))+(-(4 i+1))+(4 i+2) \\
& +(8 m+8 i+2)+(-(8 m+8 i+6))+(8 m+5-4 i)+(-(8 m+6-4 i)) .
\end{aligned}
$$

Once again a modicum of checking shows that for each vertex in $2 k C_{2 m+1} \rightarrow S$ the sequence of partial sums is made up of 8 distinct elements and so Property (iv) is satisfied.

\section{Constructing nonisomorphic embeddings}

A pair of graphs, $G_{1}$ and $G_{2}$, are isomorphic if there exists a pair of bijections, $\left(\theta_{V}, \theta_{E}\right)$ say, where $\theta_{V}: V\left(G_{1}\right) \rightarrow V\left(G_{2}\right)$ and $\theta_{E}: E\left(G_{1}\right) \rightarrow E\left(G_{2}\right)$ such that an edge $e \in E\left(G_{1}\right)$ has end vertices $x, y \in V\left(G_{1}\right)$, if and only if, $\theta_{E}(e)$ has end vertices $\theta_{V}(x), \theta_{V}(y) \in V\left(G_{2}\right)$. Two 2-cell embeddings of the complete graph $K_{n}$, say $K_{n} \rightarrow S$ and $K_{n} \rightarrow S^{\prime}$, are isomorphic if there is a bijection $\theta: V\left(K_{n}\right) \rightarrow V\left(K_{n}\right)$ such that $v_{1}, v_{2}, \ldots, v_{k}$ is a facial walk in $K_{n} \rightarrow S$, if and only if, $\theta\left(v_{1}\right), \theta\left(v_{2}\right), \ldots, \theta\left(v_{k}\right)$ is a facial walk in $K_{n} \rightarrow S^{\prime}$.

Now consider a pair of 2-cell embedding of the complete graph $K_{n}$ that are lifts of a pair of two current graphs with edge labels from the same Abelian group. In [12] Korzhik and Voss showed that a necessary condition for such a pair of embeddings to be isomorphic is that the two underlying graphs must also be isomorphic (also see [11]). This result is a key ingredient in our proofs of Theorems 3 and 4 . 
Theorem 3. For $k \geqslant 3$ and $m \geqslant 1$, there are at least $((2 m-1) !) / 2(2 m+1)=$ $2^{2 m \log _{2} m-\mathrm{O}(m)}$ nonisomorphic orientable $4 k-2 C S(8 k m+4 k+1)$ embeddings.

Theorem 4. The number of nonisomorphic orientable 8-2CS $(16 m+9)$ embeddings is at least $\sum_{s=1}^{\hat{s}} s ! p\left(2 m-5-\frac{s(s+1)}{2}, s\right)$, where $\hat{s}=\left\lfloor\frac{\sqrt{16 m-31}-3}{2}\right\rfloor$ and $p(n, s)$ denotes the number of partitions of $n$ into $s$ positive integer parts.

In order to prove Theorems 3 and 4 we first make the following observation on the proof of Theorem 2.

Observation 4.1. Let $\psi \in S_{2 m+1}$ be the permutation mapping $i$ to $i+1$ modulo $2 m+1$, for all $0 \leqslant i \leqslant 2 m$. Then, for any permutation $\phi \in S_{2 m+1}$ conjugate to $\psi$ where $\phi(2 m)=0$, suppose that we modify the construction from the proof of Theorem 2 so that the edges $f_{i}^{1}, f_{i}^{2}, \ldots, f_{i}^{2(k-1)}$ are added between vertices $v_{i}$ and $v_{\phi(i)}$ (instead of between vertices $v_{i}$ and $\left.v_{\psi(i)}\right)$ so that the edge rotations at the vertices are now

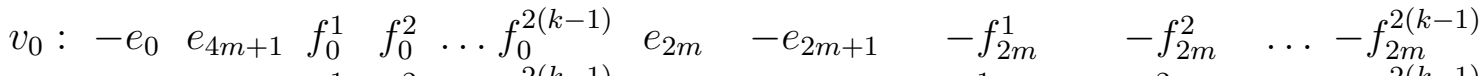

$$
\begin{aligned}
& v_{1}: e_{0} \quad-e_{1} \quad f_{1}^{1} f_{1}^{2} \ldots f_{1}^{2(k-1)} e_{2 m+1}-e_{2 m+2} \quad-f_{\phi^{-1}(1)}^{1}-f_{\phi^{-1}(1)}^{2} \cdots-f_{\phi^{-1}(1)}^{2(k-1)} \\
& v_{2}: e_{1} \quad-e_{2} \quad f_{2}^{1} \quad f_{2}^{2} \ldots f_{2}^{2(k-1)} e_{2 m+2} \quad-e_{2 m+3} \quad-f_{\phi^{-1}(2)}^{1}-f_{\phi^{-1}(2)}^{2} \cdots-f_{\phi^{-1}(2)}^{2(k-1)} \\
& v_{i}: e_{i-1} \quad-e_{i} \quad f_{i}^{1} \quad f_{i}^{2} \ldots f_{i}^{2(k-1)} e_{2 m+i}-e_{2 m+1+i}-f_{\phi^{-1}(i)}^{1} \quad-f_{\phi^{-1}(i)}^{2} \cdots-f_{\phi^{-1}(i)}^{2(k-1)} \\
& v_{2 m}: e_{2 m-1}-e_{2 m} f_{2 m}^{1} f_{2 m}^{2} \ldots f_{2 m}^{2(k-1)} e_{4 m} \quad-e_{4 m+1}-f_{\phi^{-1}(2 m)}^{1}-f_{\phi^{-1}(2 m)}^{2} \ldots-f_{\phi^{-1}(2 m)}^{2(k-1)}
\end{aligned}
$$

Then the resulting embedding still satisfies Properties (i) to (iv).

Proof. Properties (i) to (iii) follow exactly as in the proof of Theorem 2. We just need to check that all the new construction satisfies Property (iv); i.e. the sequences of partial sums at each vertex is made up of $4 k$ distinct elements. The sequence of partial sums at $v_{0}$ is unchanged so we need only to consider vertices $v_{i}$, where $1 \leqslant i \leqslant 2 m$. We will consider the two cases from the proof of Theorem 2 separately, namely $k \geqslant 3$ and $k=2$.

Suppose that $k \geqslant 3$. First note that, in the proof of Theorem 2 , for $0 \leqslant i \leqslant m$, the $(2 k+2)$-th term of the sequence of partial sums for both $v_{2 i-1}$ and $v_{2 i}$ is always $8 k m+3 k+2$. Let

$$
\begin{aligned}
& D=\{2 k-t, 8 k m+3 k+2+t: 0 \leqslant t \leqslant k-1\} ; \\
& D_{\text {odd }}=\{4 k m+6 k-4 k i, 4 k m+4 k-2 k i+t, 4 k m-k+4 k i+3: 1 \leqslant t \leqslant k-1\} ; \text { and } \\
& D_{\text {even }}=\{4 k m+4 k-4 k i, 2 k+2 k i+t, 4 k m+k+4 k i+3: 1 \leqslant t \leqslant k-1\} .
\end{aligned}
$$

Then, for all $1 \leqslant j \leqslant m$, and $1 \leqslant \ell \leqslant k-1$ the following all hold:

$$
\begin{aligned}
& 8 k m+5 k-2 k j+2-\ell, 4 k m+k+2 k j+2-\ell \notin D \text { (Claim A.1 in Appendix A); } \\
& 8 k m+5 k-2 k j+2-\ell \notin D_{\text {odd }} \text { (Claim A.2 in Appendix A); }
\end{aligned}
$$


if $i \neq j-1$, then $8 k m+5 k-2 k j+2-\ell \notin D_{\text {even }}$ (Claim A.3 in Appendix A);

if $i \neq j$, then $4 k m+k+2 k j+2-\ell \notin D_{\text {odd }}$ (Claim A.4 in Appendix A); and

$$
4 k m+k+2 k j+2-\ell \notin D_{\text {even }} \text { (Claim A.5 in Appendix A). }
$$

Hence, for the case $k \geqslant 3$, Property (iv) is satisfied and the observation follows.

Now suppose that $k=2$. This time, in the proof of Theorem 2 , for $0 \leqslant i \leqslant m$, the 6 th term of the sequence of partial sums for both $v_{2 i-1}$ and $v_{2 i-1}$ is always 1 . For all $1 \leqslant j \leqslant m$, we have the following:

$$
4 j-2,8 m+6-4 j \notin\{8 m+12-8 i, 4,8 m+8+4 i, 5,8 m+3+8 i, 1\}
$$

and

$$
4 j-2,8 m+6-4 j \notin\{8 m+8-8 i, 4,16 m+12-4 i, 5,8 m+7+8 i, 1\} .
$$

As for the previous case, the observation follows.

Let $\psi$ be defined as in Observation 4.1 and put

$$
\mathcal{S}=\left\{\phi \in S_{2 m+1}: \text { where } \phi \text { is conjugate to } \psi \text { and } \phi(2 m)=0\right\} .
$$

For each $\phi \in \mathcal{S}$, denote by $G^{\phi}$ the graph constructed in Observation 4.1. From such a graph $G^{\phi}$ a further graph $G_{\phi}$ may be constructed on the same vertex set by identifying the edge $e_{2 m+1+i}$ with the parallel edge $e_{i}$ for $0 \leqslant i \leqslant 2 m$, and by identifying together all the parallel edges $f_{i}^{j}(1 \leqslant j \leqslant 2(k-1))$ as a single edge, denoted by $f_{i}$, for $0 \leqslant i \leqslant 2 m$. We will refer to the edges $e_{i} \in E\left(G_{\phi}\right)$ as e-edges and the edges $f_{i} \in E\left(G_{\phi}\right)$ as $f$-edges. Clearly if $G^{\phi_{1}}$ and $G^{\phi_{2}}$ are isomorphic, then so are $G_{\phi_{1}}$ and $G_{\phi_{2}}$.

We now deal with the case where $k \geqslant 3$ which is somewhat simpler than the case $k=2$.

Theorem 3. For $k \geqslant 3$ and $m \geqslant 1$, there are at least $(2 m-1) ! / 2(2 m+1)=2^{2 m \log _{2} m-\mathrm{O}(m)}$ nonisomorphic orientable $4 k-2 C S(8 k m+4 k+1)$ embeddings.

Proof. Fix $k \geqslant 3$ and $m \geqslant 1$. Each $\phi \in \mathcal{S}$ may be written in cycle notation as

$$
\phi=\left(2 m, 0, a_{1}, a_{2}, \ldots, a_{2 m-1}\right),
$$

where $\left\{a_{1}, a_{2}, \ldots, a_{2 m-1}\right\}=\{1,2, \ldots, 2 m-1\}$. So the cardinality of $\mathcal{S}$ is $(2 m-1)$ !.

In $G^{\phi}$, each pair of vertices is joined by $0,2,2(k-1)$ or $2 k$ edges. Vertices joined by 2 or $2 k$ edges must be joined by $e$-edges in $G_{\phi}$ and vertices joined by $2(k-1)$ or $2 k$ edges must be joined by $f$-edges in $G_{\phi}$. Since $k \geqslant 3$, it is therefore possible to identify the cycle (of length $2 m+1$ ) of vertices joined by the $e$-edges in $G_{\phi}$.

Now suppose that $\phi_{1}, \phi_{2} \in \mathcal{S}$ and that $G^{\phi_{1}}$ and $G^{\phi_{2}}$ are isomorphic. Then there must be an isomorphism from $G_{\phi_{1}}$ to $G_{\phi_{2}}$ mapping the cycle of $e$-edges to itself. We can rotate this cycle or reflect it, so the size of an isomorphism class of $G^{\phi}$ is at most $2(2 m+1)$. Hence the number of isomorphism classes of $G^{\phi}$ is at least $(2 m-1) ! / 2(2 m+1)$. 
As mentioned at the start of this section, results from [12] show that nonisomorphic graphs $G^{\phi}$ produce nonisomorphic embeddings. So the number of nonisomorphic orientable $4 k-2 \mathrm{CS}(8 k m+4 k+1)$ embeddings is at least $(2 m-1) ! / 2(2 m+1)=2^{2 m \log _{2} m-\mathrm{O}(m)}$ as $m \rightarrow \infty$.

We next consider the case $k=2$. Our strategy is based on the following lemma

Lemma 4.1. Suppose that $m \geqslant 4$. Then two different ordered partitions of $2 m-5$ into distinct integer parts, each part of size at least 2, may be used to generate nonisomorphic graphs $G^{\phi}$. Hence the number of nonisomorphic orientable 8-2CS $(16 m+9)$ embeddings is at least the number of such partitions.

Proof. Suppose that $\mathcal{R}$ is a partition of $r=2 m-5(m \geqslant 4)$ into $s$ distinct integer parts $r_{1}, r_{2}, \ldots, r_{s}$ where $r_{1}+r_{2}+\ldots+r_{s}=r$ and $r_{i} \geqslant 2$ for $i=1,2, \ldots, s$. With each $r_{i}$ associate a graph $R_{i}$ having $r_{i}+1$ vertices and $r_{i}-1$ pairs of parallel edges having the form shown in Figure 4.

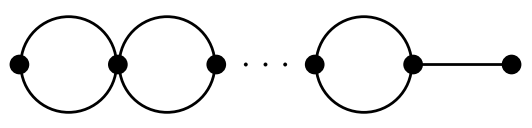

Figure 4: The graph $R_{i}$.

The graphs $R_{1}, R_{2}, \ldots, R_{s}$ may be joined end to end in order by identifying the vertex of degree 1 in $R_{i}$ with the vertex of degree 2 in $R_{i+1}$ for $1 \leqslant i \leqslant s-1$ to form a graph $R$ on $r+1$ vertices as shown in Figure 5 .

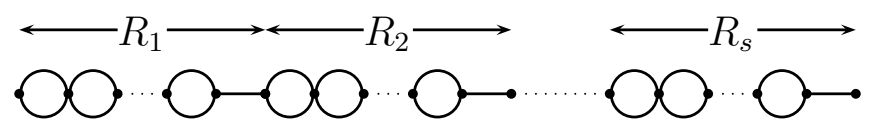

Figure 5: The graph $R$.

For any graph $G$, let $\Pi(G)$ be the subgraph induced by its parallel edges. For example, $\Pi\left(R_{i}\right)$ is isomorphic to $2 P_{r_{i}}$, where $P_{j}$ denotes a path with $j$ vertices. Similarly, $\Pi(R)$ is isomorphic to a graph with $s$ components $2 P_{r_{1}}, 2 P_{r_{2}}, \ldots, 2 P_{r_{s}}$. It follows that the partition $\mathcal{R}$ may be recovered from the graph $R$, as may the ordering of the parts.

Further edges and five new vertices are now added to $R$ to form a graph $G$ which is regular of degree 4 and whose edges may be labelled to form a graph $G_{\phi}$. The new edges and vertices are added in two stages and in such a way that no additional parallel edges are created, so that $\Pi(G)=\Pi(R)$, and the ordering of the partition $\mathcal{R}$ may also be recovered from $G$. The placement of the additional edges depends on whether $s$ is even or odd. In both cases the first stage is to take the subgraphs $R_{i}$ together in pairs, with $R_{s}$ 
left over if $s$ is odd. So, for each $i=1,2, \ldots,\left\lfloor\frac{s}{2}\right\rfloor$, join the vertex of degree 3 in $R_{2 i-1}$ to the vertex of degree 3 in $R_{2 i}$, and the vertex of degree 1 in $R_{2 i-1}$ to the vertex of degree 1 in $R_{2 i}$ (see Figure 6 where the additional edges are shown dashed). If $s$ is odd, $R_{s}$ receives no additional edges at this stage.

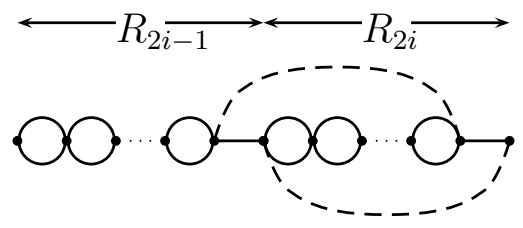

Figure 6: Additional edges between $R_{2 i-1}$ and $R_{2 i}$.

It is not possible to recover the subgraphs $R_{i}$ unambiguously from the new graph because of the additional edges. However, both the partition $\mathcal{R}$ and the ordering of this partition can still be recovered.

The second stage is to connect the end vertices of $R$, that is the vertex of degree 2 from $R_{1}$ and the vertex of degree 1 from $R_{s}$, by adding five new vertices and 12 new edges as shown in Figure 7, where six of the new edges are shown with dotted lines and six with dashed lines.

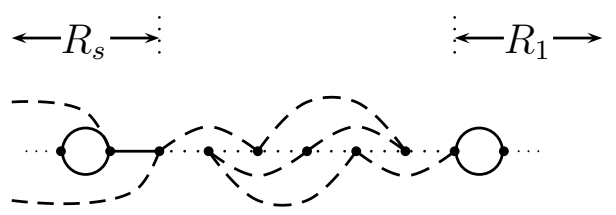

(a) $s$ even.

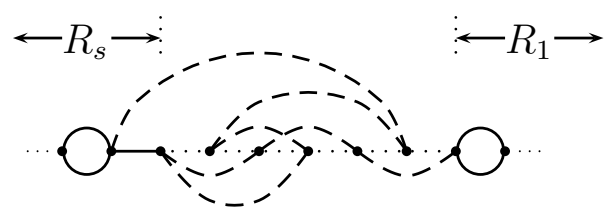

(b) $s$ odd.

Figure 7: Completing the graph $G$.

The resulting graph $G$ is regular of degree 4 . The vertex of degree 2 in the subgraph $R_{1}$ lies in a triangle in $G$ where the other two vertices of which are not in parallel edges, while the vertex of degree 3 in the subgraph $R_{s}$ does not lie in such a triangle in $G$. (This latter vertex will lie in a triangle if $r_{s}=2$ and $s$ is even, but the other two vertices of this triangle are in parallel edges.) So the vertex, say $v_{0}$, of degree 2 in $R_{1}$ may be identified and hence the direction of the ordering of the subgraphs $R_{i}$ in $G$ may be determined (that is, $\left(R_{1}, R_{2}, \ldots, R_{s}\right)$ may be distinguished from $\left.\left(R_{s}, R_{s-1}, \ldots, R_{1}\right)\right)$.

Since $r_{1} \geqslant 2$, the subgraph $R_{1}$ has a pair of parallel edges incident with $v_{0}$. Label the adjacent vertex of $R_{1}$ as $v_{2 m}$. The graph $G$ may be decomposed into two edgedisjoint cycles of length $2 m+1$ each having $v_{0}$ and $v_{2 m}$ as adjacent vertices. For example, one cycle may be taken as comprising all the dashed edges together with one edge from each pair of parallel edges, and the other cycle as comprising all the remaining edges. Label all the unlabelled vertices $v_{1}, v_{2}, \ldots, v_{2 m-1}$ so that one of these cycles is $\left(v_{0}, v_{1}, \ldots, v_{2 m}\right)$. Then label the edges of this cycle $e_{0}, e_{1}, \ldots, e_{2 m}$ so that $e_{i}$ has end 
vertices $v_{i}$ and $v_{i+1}$. The other cycle then determines a permutation $\phi \in \mathcal{S}$ such that this cycle is $\left(v_{0}, v_{2 m}, v_{\phi(2 m)}, v_{\phi^{2}(2 m)}, \ldots, v_{\phi^{(2 m-1)}(2 m)}\right)$, and the remaining unlabelled edges may be labelled $f_{0}, f_{1}, \ldots, f_{2 m}$ so that $f_{i}$ has end vertices $v_{\phi(i)}$ and $v_{i}$. The resulting labelled graph may be denoted by $G_{\phi}$. Finally each edge $e_{i}$ is replaced by parallel edges $e_{i}$ and $e_{2 m+1+i}$, and each edge $f_{i}$ is replaced by parallel edges $f_{i}^{1}$ and $f_{i}^{2}$, resulting in the graph $G^{\phi}$.

If $G^{\phi_{1}}$ and $G^{\phi_{2}}$ formed by this construction are isomorphic, then so are $G_{\phi_{1}}$ and $G_{\phi_{2}}$, and so both correspond to the same ordered partition $\mathcal{R}$. Applying Observation 4.1, the number of nonisomorphic orientable embeddings of $8-2 \mathrm{CS}(16 m+9)$ systems is therefore at least as great as the number of different ordered partitions of $2 m-5$ into distinct integer parts, each part of size at least 2 .

We now derive an estimate for the number of different ordered partitions of $n$ into distinct integer parts with each part of size at least 2 . We will denote this number by $Q(n)$. Let $q(n, s)$ be the number of partitions of the integer $n$ into $s$ distinct integer parts with each part of size at least 2. The number of ordered partitions of the integer $n$ into $s$ distinct integer parts with each part of size at least 2 is $s ! q(n, s)$, and so $Q(n)=\sum_{s} s ! q(n, s)$.

Let $p(n, s)$ denote the number of partitions of the integer $n$ into $s$ positive integer parts. If $\sum_{i=1}^{s} n_{i}=n$ where $n_{s} \geqslant n_{s-1} \geqslant \ldots \geqslant n_{1} \geqslant 1$ are integers (so that $n \geqslant s$ ), then with $n_{i}^{\prime}=$ $n_{i}+i$ we have $\sum_{i=1}^{s} n_{i}^{\prime}=n^{\prime}=n+\frac{s(s+1)}{2}$ where $n_{s}^{\prime}>n_{s-1}^{\prime}>\ldots>n_{1}^{\prime}>1$ (and $n^{\prime} \geqslant s(s+$ $3) / 2$ ). Thus every partition of $n^{\prime} \geqslant s(s+3) / 2$ into $s$ distinct integer parts where each part is of size at least 2 corresponds to a partition of $n=n^{\prime}-s(s+1) / 2$ into $s$ positive integer parts. It follows that $q(n, s)=p\left(n-\frac{s(s+1)}{2}, s\right)$. Hence $Q(n)=\sum_{s=1}^{\hat{s}} s ! p\left(n-\frac{s(s+1)}{2}, s\right)$, where $\hat{s}=\hat{s}(n)=\left\lfloor\frac{\sqrt{8 n+9}-3}{2}\right\rfloor$, so that $\frac{\hat{s}(\hat{s}+3)}{2} \leqslant \frac{1}{2}\left(\frac{\sqrt{8 n+9}-3}{2}\right)\left(\frac{\sqrt{8 n+9}+3}{2}\right)=n<\frac{(\hat{s}+1)(\hat{s}+4)}{2}$. We can now state the following result.

Theorem 4. The number of nonisomorphic orientable 8-2CS $(16 m+9)$ embeddings is at least $Q(2 m-5)=\sum_{s=1}^{\hat{s}} s ! p\left(2 m-5-\frac{s(s+1)}{2}, s\right)$, where $\hat{s}=\hat{s}(2 m-5)=\left\lfloor\frac{\sqrt{16 m-31}-3}{2}\right\rfloor$ and $p(n, s)$ denotes the number of partitions of $n$ into $s$ positive integer part.

Despite the existence of various estimates for $p(n, s)$, it has proved difficult to find a good asymptotic estimate for $Q(2 m-5)$. The following approach is very crude. Consider the partition $2+3+\ldots+\hat{s}+(\hat{s}+1)$ of $\hat{s}(\hat{s}+3) / 2$, where $\hat{s}=\hat{s}(2 m-5)=\left\lfloor\frac{\sqrt{16 m-31}-3}{2}\right\rfloor$ is as defined previously, so that $\hat{s}(\hat{s}+3) / 2 \leqslant 2 m-5$. Put $r=(2 m-5)-\hat{s}(\hat{s}+3) / 2$ and replace the term $(\hat{s}+1)$ in the partition by $(\hat{s}+1+r)$ so that it now forms a partition of $2 m-5$ into $\hat{s}$ distinct parts with each part of size at least 2 . The number of ordered partitions of $2 m-5$ into $\hat{s}$ distinct parts with each part of size at least 2 is at least $\hat{s}$ !, so $Q(2 m-5) \geqslant \hat{s} !$.

It is easy to show that if $m$ is sufficiently large then $\hat{s}>2 \sqrt{m}-3=2 \sqrt{m}\left(1-\frac{3}{2 \sqrt{m}}\right)$. 
Then, using Stirling's Theorem, we have

$$
\begin{aligned}
Q(2 m-5) & >\sqrt{2 \pi(2 \sqrt{m}-3)}\left(\frac{2 \sqrt{m}\left(1-\frac{3}{2 \sqrt{m}}\right)}{e}\right)^{2 \sqrt{m}-3} \\
& =2 \pi^{\frac{1}{2}} m^{\frac{1}{4}}\left(\frac{4 m}{e^{2}}\right)^{\sqrt{m}}\left(1-\frac{3}{2 \sqrt{m}}\right)^{2 \sqrt{m}}\left(\frac{e}{2 \sqrt{m}-3}\right)^{3}(1-\mathrm{o}(1)) \\
& =2 \pi^{\frac{1}{2}} m^{\frac{1}{4}}\left(\frac{4 m}{e^{2}}\right)^{\sqrt{m}} e^{-3} \frac{e^{3}}{8 m^{\frac{3}{2}}}(1-\mathrm{o}(1))=\frac{1}{4} \pi^{\frac{1}{2}} m^{-\frac{5}{4}}\left(\frac{4 m}{e^{2}}\right)^{\sqrt{m}}(1-\mathrm{o}(1)) .
\end{aligned}
$$

This is likely to be a considerable under-estimate for the number of embeddings.

\section{Acknowledgements}

The authors would like to thank the referee for their thoroughness and care taken in refereeing this paper.

\section{References}

[1] B. Alspach, H. Gavlas, M. Šajna, and H. Verrall, Cycle decompositions IV: Complete directed graphs and fixed length directed cycles, J. Combin. Theory Ser. A 103 (2003), 165-208.

[2] C. P. Bonnington, M. J. Grannell, T. S. Griggs, and J. Širáň, Exponential families of non-isomorphic triangulations of complete graphs, J. Combin. Theory Ser. B $\mathbf{7 8}$ (2000), no. 2, 169-184.

[3] M. N. Ellingham and C. Stephens, The nonorientable genus of joins of complete graphs with large edgeless graphs, J. Combin. Theory Ser. B 97 (2007), 827-845.

[4] M. N. Ellingham and C. Stephens, The orientable genus of some joins of complete graphs with large edgeless graphs, Discrete Math. 309 (2009), no. 5, 1190-1198.

[5] M. J. Grannell and T. S. Griggs, A lower bound for the number of triangular embeddings of some complete graphs and complete tripartite graphs, J. Combin. Theory Ser. B 98 (2008), 637-650.

[6] M. J. Grannell, T. S. Griggs and J. Širáň, Hamiltonian embeddings from triangulations, Bull. London Math. Soc. 39 (2007), 447-452.

[7] M. J. Grannell and M. Knor, A lower bound for the number of orientable triangular embeddings of some complete graphs, J. Combin. Theory Ser. B 100 (2010), 216225.

[8] T. S. Griggs and T. A. McCourt, Symmetric $n$-cycle systems on surfaces, preprint.

[9] J. L. Gross and S. R. Alpert, The topological theory of current graphs, J. Combin. Theory Ser. B 17 (1974), 218-233.

[10] J. L. Gross and T. W. Tucker, Topological Graph Theory, John Wiley, New York (1987). 
[11] V. P. Korzhik, Generating Nonisomorphic Quadrangular Embeddings of a Complete Graph, J. Graph Theory, in press.

[12] V. P. Korzhik and H-J. Voss, On the Number of Nonisomorphic Orientable Regular Embeddings of Complete Graphs, J. Combin. Theory Ser. B 81 (2001), no. 1, 58-76.

[13] G. Ringel, Map Color Theorem, Springer-Verlag, New York (1974).

[14] J. W. T. Youngs, The mystery of the Heawood conjecture, in Graph Theory and its Applications, Academic Press, New York (1970), 17-50. 


\section{A Observation 4.1}

Suppose that $m \geqslant 1$ and $k \geqslant 3$. For $0 \leqslant i \leqslant m$, in the proof of Observation 4.1 , the following sets are defined.

$$
\begin{aligned}
& D=\{2 k-t, 8 k m+3 k+2+t: 0 \leqslant t \leqslant k-1\} ; \\
& D_{\text {odd }}=\{4 k m+6 k-4 k i, 4 k m+4 k-2 k i+t, 4 k m-k+4 k i+3: 1 \leqslant t \leqslant k-1\} ; \text { and } \\
& D_{\text {even }}=\{4 k m+4 k-4 k i, 2 k+2 k i+t, 4 k m+k+4 k i+3: 1 \leqslant t \leqslant k-1\} .
\end{aligned}
$$

Claim A.1. For all $1 \leqslant j \leqslant m$ and $1 \leqslant \ell \leqslant k-1 ; 8 k m+5 k-2 k j+2-\ell, 4 k m+k+$ $2 k j+2-\ell \notin D$.

Proof. Let $0 \leqslant t \leqslant k-1$. Suppose that, for some $1 \leqslant j \leqslant m$ and $1 \leqslant \ell \leqslant k-1$; $\{8 k m+5 k-2 k j+2-\ell, 4 k m+k+2 k j+2-\ell\} \cap D \neq \emptyset$. Then

$$
\begin{aligned}
& 8 k m+5 k-2 k j+2-\ell=2 k-t, \text { so } 8 k m+3 k+2+t=2 k j+2 k+\ell \leqslant 2 k m+3 k-1 ; \text { or } \\
& 8 k m+5 k-2 k j+2-\ell=8 k m+3 k+2+t, \text { so } 2 k=t+2 k j+\ell, \text { hence } 2 k \mid(t+\ell) ; \text { or } \\
& 4 k m+k+2 k j+2-\ell=2 k-t, \text { so } 4 k m+2 k j+2+t=k+\ell \leqslant 2 k-1 ; \text { or } \\
& 4 k m+k+2 k j+2-\ell=8 k m+3 k+2+t, \text { so } 2 k m \geqslant 2 k j=4 k m+2 k+t+\ell .
\end{aligned}
$$

Thus, in all four cases, we have a contradiction.

Claim A.2. For all $1 \leqslant j \leqslant m$ and $1 \leqslant \ell \leqslant k-1 ; 8 k m+5 k-2 k j+2-\ell \notin D_{\text {odd }}$.

Proof. Let $0 \leqslant t \leqslant k-1$. Suppose that, for some $1 \leqslant i, j \leqslant m$ and $1 \leqslant \ell \leqslant k-1$; $8 k m+5 k-2 k j+2-\ell \in D_{\text {odd }}$. Then

$$
\begin{aligned}
& 8 k m+5 k-2 k j+2-\ell=4 k m+6 k-4 k i, \text { so } 4 k m+4 k i+2=k+2 k j+\ell \leqslant 2 k m+2 k-1 ; \text { or } \\
& 8 k m+5 k-2 k j+2-\ell=4 k m+4 k-2 k i+t, \text { so } 4 k m+k+2 k i+2=2 k j+\ell+t \leqslant \\
& 2 k m+2 k-2 ; \text { or } \\
& 8 k m+5 k-2 k j+2-\ell=4 k m-k+4 k i+3, \text { so } 4 k m+6 k=4 k i+2 k j+\ell+1, \text { hence } \\
& 2 k \mid(\ell+1) .
\end{aligned}
$$

Thus, in all three cases, we have a contradiction.

Claim A.3. For all $1 \leqslant j \leqslant m$ and $1 \leqslant \ell \leqslant k-1$; if $i \neq j-1$, then $8 k m+5 k-2 k j+2-\ell \notin$ $D_{\text {even }}$.

Proof. Let $0 \leqslant t \leqslant k-1$. Suppose that, for some $1 \leqslant i, j \leqslant m$ where $i \neq j-1$ and $1 \leqslant \ell \leqslant k-1 ; 8 k m+5 k-2 k j+2-\ell \in D_{\text {even }}$. Then

$$
\begin{aligned}
& 8 k m+5 k-2 k j+2-\ell=4 k m+4 k-4 k i, \text { so } 4 k m+k+4 k i+2=2 k j+\ell \leqslant 2 k m+k-1 ; \text { or } \\
& 8 k m+5 k-2 k j+2-\ell=2 k+2 k i+t, \text { so } 8 k m+3 k+2=2 k i+2 k j+t+\ell \leqslant \\
& 4 k m+2 k-2 ; \text { or }
\end{aligned}
$$


$8 k m+5 k-2 k j+2-\ell=4 k m+k+4 k i+3$, so $4 k m+4 k=4 k i+2 k j+\ell+1$, hence $2 k \mid(\ell+1)$.

Thus, in all three cases, we have a contradiction.

Claim A.4. For all $1 \leqslant j \leqslant m$ and $1 \leqslant \ell \leqslant k-1$; if $i \neq j$, then $4 k m+k+2 k j+2-\ell \notin$ $D_{\text {odd }}$.

Proof. Let $0 \leqslant t \leqslant k-1$. Suppose that, for some $1 \leqslant i, j \leqslant m$ where $i \neq j$ and $1 \leqslant \ell \leqslant k-1 ; 4 k m+k+2 k j+2-\ell \in D_{\text {odd }}$. Then

$$
\begin{aligned}
& 4 k m+k+2 k j+2-\ell=4 k m+6 k-4 k i, \text { so, as } i \neq j, 8 k+2 \leqslant 2+2 k j+4 k i= \\
& 5 k+\ell \leqslant 6 k-1 ; \text { or } \\
& 4 k m+k+2 k j+2-\ell=4 k m+4 k-2 k i+t, \text { so, as } i \neq j, 6 k+2 \leqslant 2+2 k j+2 k i= \\
& 3 k+t+\ell \leqslant 5 k-2 ; \text { or } \\
& 4 k m+k+2 k j+2-\ell=4 k m-k+4 k i+3, \text { so } 2 k+2 k j=4 k i+\ell+1, \text { hence } 2 k \mid(\ell+1) .
\end{aligned}
$$

Thus, in all three cases, we have a contradiction.

Claim A.5. For all $1 \leqslant j \leqslant m$ and $1 \leqslant \ell \leqslant k-1 ; 4 k m+k+2 k j+2-\ell \notin D_{\text {even }}$.

Proof. Let $0 \leqslant t \leqslant k-1$. Suppose that, for some $1 \leqslant i, j \leqslant m$ and $1 \leqslant \ell \leqslant k-1$; $4 k m+k+2 k j+2-\ell \in D_{\text {even }}$. Then

$$
\begin{aligned}
& 4 k m+k+2 k j+2-\ell=4 k m+4 k-4 k i, \text { so } 4 k i+2 k j+2=3 k+\ell \leqslant 4 k-1 \text {; or } \\
& 4 k m+k+2 k j+2-\ell=2 k+2 k i+t, \text { so } 4 k m+2 k j+2=k+2 k i+t+\ell \leqslant 2 k m+3 k-2 \text {; or } \\
& 4 k m+k+2 k j+2-\ell=4 k m+k+4 k i+3, \text { so } 2 k j=4 k i+\ell+1, \text { hence } 2 k \mid(\ell+1) .
\end{aligned}
$$

Thus, in all three cases, we have a contradiction. 